\title{
Referral paths in the U.S. physician network
}

\author{
Chuankai An ${ }^{*}$ (1) A. James O'Malley² and Daniel N. Rockmore $1,3,4$
}

\author{
*Correspondence: \\ chuankai@cs.dartmouth.edu \\ 'Department of Computer Science, \\ Dartmouth College, 03755 Hanover, \\ $\mathrm{NH}$, USA \\ Full list of author information is \\ available at the end of the article
}

\begin{abstract}
In this paper, we analyze the millions of referral paths of patients' interactions with the healthcare system for each year in the 2006-2011 time period and relate them to U.S. cardiovascular treatment records. For a patient, a "referral path" records the chronological sequence of physicians encountered by a patient (subject to certain constraints on the times between encounters). It provides a basic unit of analysis in a broader referral network that encodes the flow of patients and information between physicians in a healthcare system. We consider referral networks defined over a range of interactions as well as the characteristics of referral paths, producing a characterization of the various networks as well as the physicians they comprise. We further relate these metrics and findings to outcomes in the specific area of cardiovascular care. In particular, we match a referral path to occurrences of Acute Myocardial Infarction (AMI) and use the summary measures of the referral path to predict the treatment a patient receives and medical outcomes following treatment. Some referral path features are more significant with respect to their ability to boost a tree-based predictive model, and have stronger correlations with numerical treatment outcome variables. The patterns of referral paths and the derived informative features illustrate the potential for using network science to optimize patient referrals in healthcare systems for improved treatment outcomes and more efficient utilization of medical resources.
\end{abstract}

Keywords: Network science, Big data, Health record analysis, Social network analysis, Predictive modeling

\section{Introduction}

A well-designed healthcare system is a crucial element of a well-functioning society, and the ways in which information and resources flow in such a system are key determinants of its efficacy. Patient referrals serve as a useful and measurable proxy for communication and collaboration between physicians in different specialties (Burns and Muller 2008). Physicians refer a patient to other physicians who will either be within or outside of their own hospital, generally (although not exclusively) for considerations relevant to the care of patients. To that end, reasons range from the need for specialized care to addressing problems of overcrowding (and thus postponing care). In aggregate, the sequence of referrals - a referral path - for a given patient over the course of treatment for a given concern is thus an important record of a focused interaction of a patient with the healthcare system. It represents a collection of pairwise and possibly group information sharing opportunities about treatment among two or even a team of physicians involved in the treatment of a patient.

(c) The Author(s). 2018 Open Access This article is distributed under the terms of the Creative Commons Attribution 4.0 International License (http://creativecommons.org/licenses/by/4.0/), which permits unrestricted use, distribution, and reproduction in any medium, provided you give appropriate credit to the original author(s) and the source, provide a link to the Creative Commons license, and indicate if changes were made. 
The language (and mathematics) of network science is well-adapted to the study of such discretized and localized information and resource flow. In the particular case of healthcare we use network models and measures as a way of understanding patient care, healthcare resource allocation and treatment efficiency. To that end the referral of a patient by physician $A$ to physician $B$ is naturally represented as a directed edge from one network node to another. A referral path also stores the date of the visit and interactions between a patient and each physician on the path. Possibly because of specialty, different physicians might spend uneven amounts of time and effort (e.g., as measured by the relative value unit or " $\mathrm{RVU}$ ") during a typical encounter with a patient. We describe the referral path in terms of multiple features (e.g., time between initial and final encounters or average RVU). Domains of investigation can range from the network of physicians in or attributed to a hospital, the Hospital Referral Region (HRR), or the entire United States referral network. A range of choices for edge weights can articulate different properties of these interactions. Given groups of referral network structural measures and referral path features, multilevel regression models and classification methods in machine learning have the potential to reveal relationships between the organization of patient flow in the healthcare system and the well-being of patients, and with this, insights into improving efficacy and resource allocation for our healthcare system.

In earlier work (An et al. 2018) we presented an analysis of the U.S. patient referral network, subjecting it and its HRR and state-level subnetworks to a range of network analyses to uncover their large-scale network structure. This work built on earlier work (Landon et al. 2012; Mandl et al. 2014; Lee et al. 2011; Lomi et al. 2014; Donker et al. 2010; Shea et al. 1999). Example results include the existence of power laws in degree distributions, "small-world" and core-periphery structures, and a statistical analysis of the motif structures in these networks. A suite of regressions also uncovered interesting relationships among the various network metrics. In this paper we study the more fine-scale patterns to be found in the consideration of the referral paths and importantly link these statistics to treatment outcomes in the particular setting of cardiovascular disease. While referral path and referral information generally has been ignored as a factor in the important problem of treatment outcome prediction, the predictive value of other kinds of data have been studied. In (Fiterau et al. 2017), researchers applied deep neural networks to time series of sensory data to predict other diagnoses. Several works (Liaw 2009; Ellis et al. 2008; Ball et al. 2014) in medical research mainly focused on variables from clinical medical tests and used standard statistical analysis techniques to make inferences about the relationships of treatments to outcomes.

Prior studies related to referral paths have been limited in terms of the range of health records studied (Uddin et al. 2013; Uddin 2016). In this paper we introduce new metrics related to the study of referral paths and are able to compute detailed network measures in a much larger dataset (the TDI ${ }^{2}$ dataset) of cardiovascular disease treatment, ranging from a local hospital or HRR to the current national referral network. Aggregating the data from thousands of local hospitals and hundreds of HRRs, we use statistical methods to validate the general patterns of referral paths and referral networks. We characterize the dynamics of changes of node position and type among all physicians on a referral path. In the case of cardiovascular treatment, we find evidence of key roles on a referral path, especially for the physicians with a specialty of cardiovascular and internal medicine. We also validate the prevalence of patterns of referrals indicating that physicians work with 
their professional acquaintances when choosing the target of a referral, i.e., regularly send patients to the physicians who have many common collaborators. We then apply classification models to the cardiovascular referral network measures and referral path features to predict teaching status of a hospital and a patient's treatment outcome (e.g., indicator of death within 1 year after treatment). Our considerations of networks and referral paths for cardiovascular treatment could clearly be adapted for other contexts. More specifically, given patient referral records tied to a different disease state, the metrics and methodologies we introduce here (e.g., the feature and pattern mining, model selection, analysis, etc.) could be directly adapted. In addition, our study has implications for research about a generalized notion of a referral path in such contexts as information flow in online media or social networks.

Some specific contributions of our work include:

- Novel definition of the health records-based referral path as well as novel definition of salient features for referral paths generated from both network science and time series analysis.

- Quantification of a physician's position using centrality and other measures in the U.S. national cardiovascular referral network with the help of techniques specific to big data that are necessary for overcoming the infeasibility of using traditional algorithms for calculations at scale.

- Investigation of the patterns of millions of referral paths in the referral network, which are validated by statistical tests.

- Effective classification and regression models derived from novel referral path features and referral networks that distinguish (a) teaching status of a hospital and (b) patient treatment outcomes. These models pick up key predictors among network measures relevant to the optimization of an effective healthcare system.

\section{Materials, notation, and methodology}

\section{Materials}

We used Medicare beneficiary claims data for all patients diagnosed with cardiovascular disease in the U.S. during 2006-2011 to build referral paths and networks of the US healthcare system. Here cardiovascular disease means that the patient suffers from arrhythmia, congestive heart failure, coronary-heart disease or peripheral vascular disease in the diagnostic codes of Medicare claims. This dataset is of interest for several reasons. It is on the one hand a kind of network "big data" (as we will see, the data produce networks on hundreds of thousand of nodes and millions of edges) in a research area (healthcare) where traditionally data analysis has not been accomplished at this scale (i.e., related work considers data at the level of the health care unit - e.g., hospital - or a local region). In our previous work (An et al. 2018) related to national networks we had much less metadata so that our work was more descriptive. This richer data enables us to begin to create more interesting methodologies for this kind of data. In particular, by focusing on the part of the national dataset related to a disease diagnosis, we can begin to articulate and build out methodologies that relate to outcomes. With the exception of patients dually eligible for Medicare and Medicaid, these data contain a record of each physician encounter of each Medicare patient. Each such record contains the patient or "beneficiary" (Bene) identification (ID) number, physician National Provider Identification (NPI) number, visit date, RVU associated with the visit and other details ${ }^{3}$. Since the NPI numbers for all 
physicians changed in 2007, some of the analysis we perform only obtains for the interval 2007-2011. Although claims data and other sources of patient-physician encounters has been previously used to form physician networks (An et al. 2018; Landon et al. 2012; Mandl et al. 2014; Lomi et al. 2014; Shea et al. 1999), in this paper we apply a more nuanced approach.

At the heart of this is the notion of a "referral from physician $A$ to physician $B$ ", which we define as the event that a patient encounters physician $B$ within 30 days of encountering physician $A$ (and encounters no other physician in between those times). The "referral path" is a maximal sequence of referrals, assumed to embody the team of physicians involved in the treatment of a patient over the course of a given episode of illness. A referral path might connect physicians in different areas. Since each visiting record includes the HRR and hospital where a physician is working or attributed on the basis of where most of their patients are hospitalized (Bynum et al. 2007), we can categorize referral paths as purely intra- versus inter-hospital or HRR. Similarly, the various network measures to be able to be evaluated for each HRR or hospital level (PHN) subnetwork. In this paper we will be primarily interested in cardiovascular referral networks, since the raw records of patient-physician visits are specific to cardiovascular disease treatment in U.S.

\section{Referral path}

The relationship between patients and physicians is naturally represented as a bipartite graph. In Fig. 1, several edges connect two patients $(\alpha$ and $\beta$ ) to some physicians whom they have visited. Patient $\alpha$ visits four physicians in the sequence $(A, B, C, D)$ and patient $\beta$ visits $B$ and $C$. By sorting the four physicians according to the date of patient $\alpha$ 's visit, we recover a sequence of four physicians reflecting the sequence of encounters. In this paper, we define a patient referral path as a sequence of physicians whom the patient encounters in chronological order. If a patient encounters a physician followed by another within a threshold of 30 days (i.e., a referral exists), we assume there is an information exchange opportunity between the two physicians.

\section{Referral network and computation of edge weights}

The referral network (over a given time period) is a directed network with node set given by the physicians present in the database over a fixed time period. If physician $A$ refers at least one patient to physician $B$, this is represented by a directed edge from $A$ to $B$. Given all referrals over a year, we are able to build the U.S. national patient referral network of US

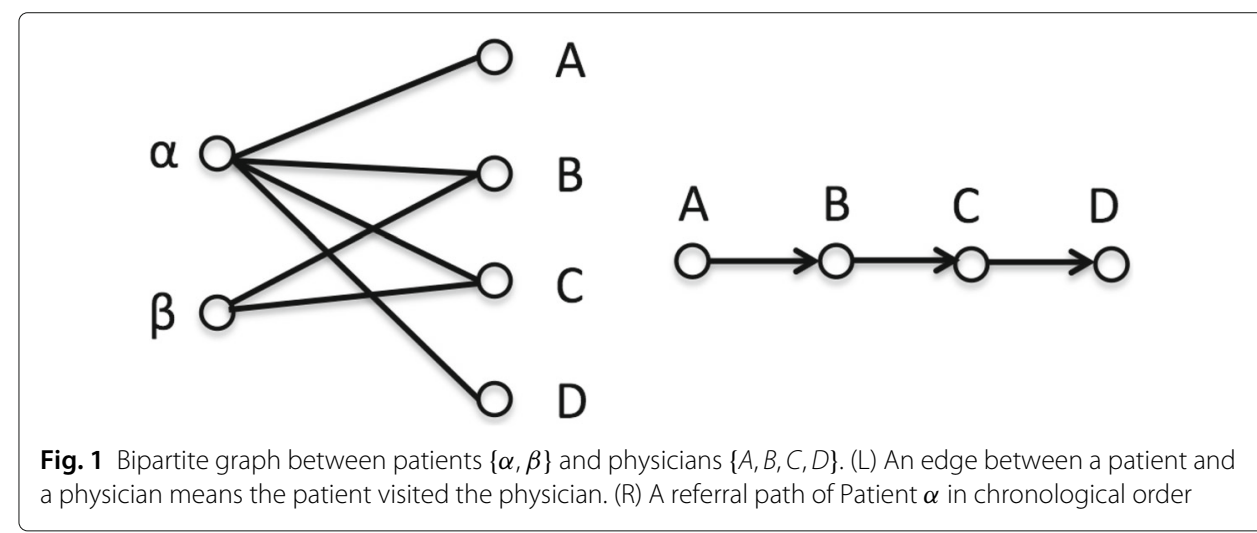


physicians. In this paper, we mainly investigate micro-patterns of referral paths for each patient in HRR/PHN referral networks, while our prior work (An et al. 2018) introduces macro-patterns derived from directed national, HRR, and state referral sub-networks. Herein, most of the network measures are also derived from directed referral networks, except a few measures from the corresponding undirected networks, such as diameter, clustering coefficient and giant component.

Edges can be weighted in a variety of ways. A simple unweighted edge (i.e., edge weight equal to 1) denotes simply a connection. More information is added if we use other natural metrics such as the number of referrals or the geometric mean of RVUs. A novel metric that we define here is the "ranking based weight": Let the vector $r=(1,2, \ldots, n)$ denote the chronological "ranks" 4 of the encounters on a referral path consisting of $n$ physicians. In this case for a given physician $A$, let $n_{A}$ denote the number of encounters for physician $A$ on the referral path, and let $r_{A}$ be the sub-list of the ranks of the encounters with a physician in the referral path (so, if $A$ was encountered on the first and last visits only, then $\left.r_{A}=(1, n)\right)$. In this way, $n_{A}$ is the length of the $r_{A}$. The flow of patients from physician $A$ to physician $B$ is then given by

$$
f_{A B}=\frac{\sum_{i<j} I\left(r_{A i}<r_{B j}\right)}{n_{A} n_{B}}
$$

and from $B$ to $A$ by

$$
f_{B A}=\frac{\sum_{i<j} I\left(r_{A i}>r_{B j}\right)}{n_{A} n_{B}} .
$$

To compute the ranking based weight of an edge, we compute a weighted sum of the patient ranking index flow in each referral path $p$ containing both physician $A$ and $B$. A referral path $p$ might include multiple physicians, but the flow of patients in the referral path between physician $A$ and $B$ only relates to their sub-vectors $r_{A}$ and $r_{B}$, without any impact from a third physician. The function of Eq. (1) lies in [0, 1], and under the assumption of a stationary model of doctor's visit occurrence it will converge to a constant as $n_{A}$ and $n_{B}$ go to infinity, but we would like to account for the length of each referral path, so we add $n_{A p}$ and $n_{B p}$ and weight the contribution from each referral path by its geometric mean in Eq. (3).

$$
w_{A B}=\sum_{p}\left(n_{A p} n_{B p}\right)^{1 / 2} f_{A B p}
$$

To sum up, Table 1 shows an interim step of the data processing process with the format of input data and the output of referral paths/networks.

\section{Referral path features}

In (An et al. 2018) we introduce the use of various basic network measures for the study of patient referral networks and uncover macro-level network structures including general patterns of "power law" in degree distribution, "small-world" structure, core-periphery structure, and the existence of a "gravity law" in a state-level referral traffic map. In this paper we focus on the referral path and to that end, introduce some metrics that get at the diversity of a referral path. Denote the number of visits on a referral path as $N$, the $i$ th node on a referral path as $P_{i}$, the date of the encounter with the $i$ th node as $T_{i}, 1 \leqslant i \leqslant N$. With this notation we make the following definitions and illustrate them using the example 
Table 1 Example pipeline of data processing from raw patient-physician encounter records to referral paths and edges of referral network

\begin{tabular}{|c|c|c|}
\hline \multicolumn{3}{|c|}{ (a) Raw visiting records } \\
\hline Patient & Physician & date;HRR;HRRcity;state;zipcode;workRVU;specialty;PHN;teaching type; etc. \\
\hline$\alpha$ & A & 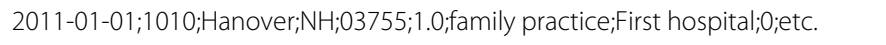 \\
\hline$\alpha$ & B & 2011-01-10;1020;Boston;MA;02101;3.0;internal medicine;Second hospital;1; etc. \\
\hline$\alpha$ & C & 2011-02-01;1050;New York;NY;10021;4.0;cardiology;Third hospital;1;etc. \\
\hline$\beta$ & B & 2011-03-01;1012;Lebanon;NH;03784;2.0;family practice;Fourth hospital;0;etc. \\
\hline$\beta$ & C & 2011-03-20;1022;Newton;MA;02461;5.0;vascular surgery;Fifth hospital;1; etc. \\
\hline \multicolumn{3}{|c|}{ (b) Referral path } \\
\hline Patient & & Node(date;\#visiting records; RVU),divided by "->" \\
\hline$\alpha$ & & $A(2011-01-01 ; 1.0,1.0)->B(2011-01-10 ; 1.0 ; 3.0)->C(2011-02-01 ; 1.0 ; 4.0)$ \\
\hline$\beta$ & & $B(2011-03-01 ; 1.0 ; 2.0)->C(2011-03-20 ; 1.0 ; 5.0)$ \\
\hline \multicolumn{3}{|c|}{ (c) Edges in the national referral network with the weights over all referral paths } \\
\hline Directed edge & & Weights of an edge \\
\hline$A->B$ & & $3 ; 4 ; 4.82 ; 12.14 ; 23.42$ \\
\hline$B->C$ & & $5 ; 5 ; 5.12 ; 12.32 ; 18.22$ \\
\hline
\end{tabular}

in Fig. 2 (note that in Fig. 2, the nodes corresponding to the physicians are color-coded according to some affiliation datum - e.g., HRR or hospital):

- Path length. The total number of physicians on a referral path. A physician could be counted multiple times if the patient visits the physician again. It is 5 in Fig. 2.

- Average time gap between referrals on the referral path: $\frac{T_{N}-T_{1}}{N-1}$.

- Time range. $T_{N}-T_{1}$. It is the gap between the last visit and the first.

- Recurrence. A binary variable recording whether there exists $i, j$, with $1 \leqslant i<j \leqslant N$, and $P_{i}=P_{j}$. It is true (set to "1") in Fig. 2 because of multiple occurrences of physicians $A$ and $B$.

- Number of nodes before recurrence. This is defined as $\min \{j\}-1$, where $(i, j)$ satisfy the above recurrence condition. It refers to the first reappearance of a node. In our example, it is 3 since the first three nodes $A, B, C$ are different from each other before the first duplicate node, $B$.

- Physician distribution entropy. This is the standard probabilistic definition of entropy $\left(-\sum_{x} p(x) \log _{2}(x)\right)$ derived here from the physician occurrence probability over the path. In Fig. 2, the frequencies of $A, B, C$ are 2,2,1 respectively. The physician distribution entropy of the related probability distribution $(0.4,0.4,0.2)$ is 1.522 .

- Hospital distribution entropy. The entropy of the derived physicians' hospital distribution is another feature of diversity. Since we assume $A$ and $C$ are from the same hospital, the frequency distribution is $(3,2)$ and the corresponding entropy is 0.971 .

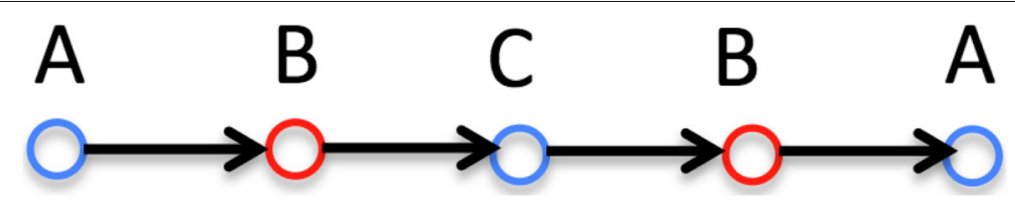

Fig. 2 An example referral path with three physicians $A, B, C$. The patient visits them five times. Physicians $A$ and $C$ are from the same HRR/hospital in blue, while physician $B$ is from another HRR/hospital in red 
- HRR distribution entropy. The entropy of the physicians' HRR probability is another feature of diversity. It is the same value as PHN distribution entropy under the assumption that $A$ and $C$ are in the same HRR.

- Main hospital. It is a derived referral path feature of the hospital in which the most physicians on the referral path are working. It is the hospital with $A$ and $C$ in Fig. 2.

- Main or dominant HRR. The HRR in which the most physicians are working. It is the HRR with $A$ and $C$ in Fig. 2.

- Number of pairs of nodes with reciprocal referrals on a referral path. $\sum_{i, j} 1\left(1 \leqslant i<j \leqslant T-1, P_{i}=P_{j+1}, P_{i+1}=P_{j}\right)$. There are two pairs of nodes $(A, B)$ and $(B, C)$ which have such reciprocal relations.

\section{Node position features}

In a referral network, metrics related to node characteristics correspond to metrics of physician "importance". Meaningful examples include local clustering coefficient, betweenness centrality, closeness centrality, eigenvector centrality, PageRank centrality (Page et al. 1999), core-periphery score (Rombach et al. 2017). In addition, we adapt the notion of h-index to the patient referral network (Hirsch 2005). For a node in the national referral network, consider the array of indegrees for all nodes which refer patients to the node, then count the h-index of the indegree array, which means $h$ referral source nodes have at least $h$ indegree in the array.

Here are some of the features describing node position that are relevant to the context of referral paths.

- Number of paths that contain the node.

- Number of paths where the node is the initial visit. In Fig. 2, physician $A$ is the first node.

- Number of paths where the node is the final visit. In Fig. 2, physician $A$ is the end node.

- Average index of the first-time occurrence in all paths. In Fig. 2, the index of first-time occurrence for nodes $A, B, C$ is $1,2,3$, respectively, so we can take the average over all referral paths.

- Number of paths where the node occurs multiple times. In Fig. 2, nodes $A$ and $B$ occur twice.

- Number of cross-HRR referrals proposed by the node. In Fig. 2, given the assumption that nodes $A$ and $C$ are from the same HRR, node $A$ sends patients to node $B$ in another HRR. Nodes $B$ and $C$ also form an edge that spans HRRs.

- Number of cross-hospital referrals proposed by the node. In Fig. 2, given the assumption that nodes $A$ and $C$ are from the same PHN, node $A$ sends patients to node $B$ in another hospital. The same is true of nodes $B$ and $C$.

\section{Results}

In this project, we process raw patient-physician encounter records, build referral paths/networks and derive the following patterns in Python, with the help of NetworkX (NetworkX). We build the machine learning programs for treatment outcome prediction with scikit-learn (Scikit-learn: Machine learning in Python), and implement statistical tests and regression models in $\mathrm{R}$. 


\section{National, HRR and PHN network measures}

Table 2 shows several measures (see (An et al. 2018)) over the period 2006-2011 of the big national referral network with millions of edges. Generally speaking, the national referral network restricted to U.S. cardiovascular treatment reveals macro-level patterns found in the larger "all-inclusive" U.S. referral network (An et al. 2018). For this dataset, the increased number of nodes and edges in the national referral network might be a result of the elderly population growing in number across time. We also compute those network measures for the U.S. cardiovascular treatment network restricted to the 300+ HRR and 4,800+ hospital subnetworks. In this way we characterize the standing of the physicians in a referral path nationally, locally, and institutionally. Unless otherwise noted, when we refer to a referral network in this paper we mean the US cardiovascular care patient referral network to which our methods are applied.

\section{Referral path features}

Table 3 describes features of millions of referral paths over 2006-2011. The average duration of each referral path is roughly 25 days (avg time range) and comprises about four nodes (avg length). About one-third of referral paths have a node which the "defining patient" visits multiple times. The distribution of the referral paths when weighted by hospital entropy is more diverse than when weighted by HRR entropy, which implies that a patient will more likely visit multiple hospitals in the same HRR than to have multiple visits in different regions (HRRs). Close to half of the pairs on a given referral path are reciprocating.

\section{Patterns of referral paths}

In addition to the basic overall features for all referral paths, we explore other patterns from other perspectives.

Index on Referral Path vs. Node Position in Network Corresponding "node position sequences" encode the ways in which a patient navigates along physicians in terms of the physician position of importance in the referral network. Here we consider the node position sequence with respect to five node position measures in the national referral network: clustering coefficient, betweenness centrality, eigenvector centrality, PageRank centrality and h-index. Figure 3 shows an observed node position sequence represented

Table 2 Some national referral network measures in 2006-2011

\begin{tabular}{lllllll}
\hline Year & 2006 & 2007 & 2008 & 2009 & 2010 & 2011 \\
\hline \# nodes & 272353 & 296008 & 313051 & 323042 & 334452 & 347586 \\
\# edges & 5708791 & 5948185 & 6313136 & 6544847 & 6785594 & 7047586 \\
Exponent of indegree power law & 3.08 & 2.80 & 1.55 & 2.76 & 1.54 & 2.74 \\
p-value of indegree power law test & 0.97 & 0.89 & 0.21 & 0.85 & 0.22 & 0.82 \\
Exponent of outdegree power law & 3.01 & 2.69 & 2.71 & 2.66 & 2.56 & 2.68 \\
p-value of outdegree power law test & 0.9 & 0.94 & 0.93 & 0.96 & 0.91 & 0.93 \\
Size of the largest connected component & 271898 & 295405 & 312412 & 322452 & 333727 & 346711 \\
(in, in) degree assortativity & -0.094 & -0.088 & -0.084 & -0.085 & -0.083 & -0.084 \\
Self in/out degree correlation & 0.983 & 0.982 & 0.983 & 0.983 & 0.983 & 0.984 \\
Reciprocity of \#referral & 0.878 & 0.890 & 0.896 & 0.901 & 0.902 & 0.896 \\
\hline
\end{tabular}


Table 3 Overall statistics of all referral paths in 2006-2011

\begin{tabular}{lllllll}
\hline Year & 2006 & 2007 & 2008 & 2009 & 2010 & 2011 \\
\hline \#referral paths & $4.44 \mathrm{M}$ & $4.45 \mathrm{M}$ & $4.54 \mathrm{M}$ & $4.59 \mathrm{M}$ & $4.63 \mathrm{M}$ & $4.66 \mathrm{M}$ \\
Avg length & 3.850 & 3.907 & 3.983 & 4.023 & 4.061 & 4.115 \\
Avg gap for a referral & 8.509 & 8.506 & 8.369 & 8.352 & 8.230 & 8.060 \\
Avg time range & 24.247 & 24.727 & 24.969 & 25.245 & 25.192 & 25.109 \\
Percent of paths with recurrent nodes & 33.418 & 32.879 & 32.836 & 32.784 & 32.573 & 32.301 \\
Avg \#nodes before recurrence & 4.087 & 4.130 & 4.179 & 4.196 & 4.223 & 4.271 \\
Avg physician entropy & 1.400 & 1.410 & 1.423 & 1.427 & 1.436 & 1.448 \\
Avg hospital entropy & 0.475 & 0.473 & 0.476 & 0.459 & 0.480 & 0.481 \\
Avg HRR entropy & 0.107 & 0.109 & 0.108 & 0.105 & 0.112 & 0.116 \\
Avg bidirectional pairs in a path & 0.450 & 0.455 & 0.465 & 0.474 & 0.476 & 0.479 \\
\hline
\end{tabular}

by the local clustering coefficient of each node. After classical seasonal decomposition (Classical seasonal decomposition by moving averages) by moving averages on the sequence, the seasonal component tends to fluctuate, which suggests that physicians in the core and periphery parts appear alternately on the referral path.

Denote the $N$ physicians on a referral path as $P=\left(P_{1}, P_{2}, \ldots P_{N}\right)$ and the node position value of $P_{i}$ as $C_{i}$, so that the corresponding node position sequence can be denoted as

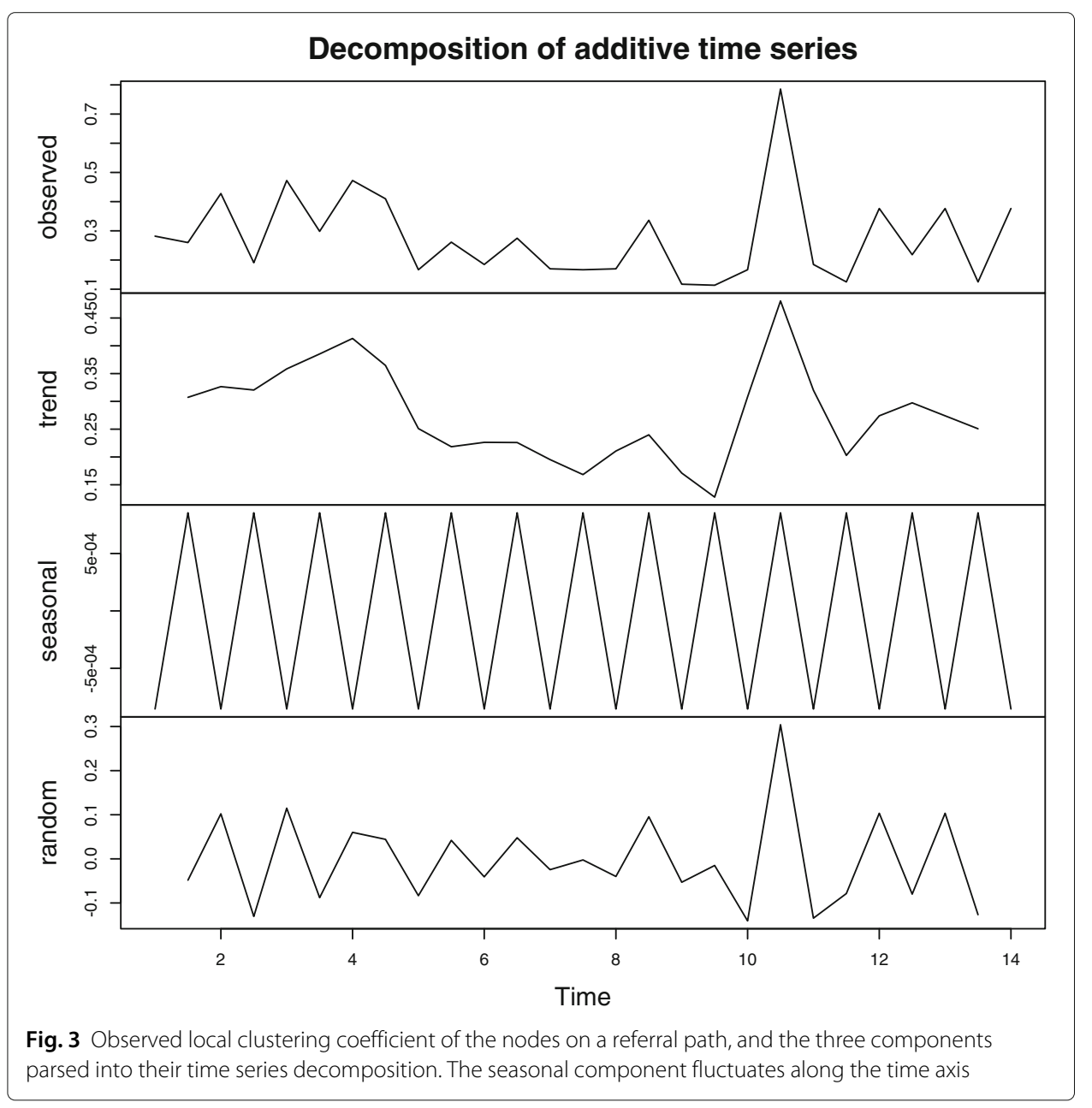


$C=\left(C_{1}, C_{2}, \ldots C_{N}\right)$. Then the number of changes in trend $\sum_{i=2}^{N-1} 1\left(\left(C_{i}-C_{i-1}\right)\left(C_{i+1}-\right.\right.$ $\left.C_{i}\right)<0$ ) counts the change of sgn (positive, negative) of the difference in the centrality of successive providers on referral path $P_{i}, 2 \leq i \leq N-1$. The event $\left(C_{i}-C_{i-1}\right)\left(C_{i+1}-\right.$ $\left.C_{i}\right)<0$ is defined as a change point. For each node in the middle of a referral path, if the neighboring nodes and itself satisfy the condition, it contributes one to the number of change points.

Table 4 shows the percentage of change points in terms of five kinds of node position measures in 2007-2011. In most cases, a patient will alternate visits between a physician with a larger centrality measure and one with smaller centrality measure. The pattern is stable in different years with all node centrality measures, which suggests that some core physicians in the national referral network help to link some physicians with fewer referrals for the patient's treatment.

The fluctuation suggests that on a referral path some physicians with relatively larger centrality measure might diagnose the disease and organize the referral path by referring the patient to nodes with lower centrality. This is the role that has been envisioned for primary care physicians in the health care system and prior network analyses (Barnett et al. 2012) have found that the more prominent (i.e., central) primary care physicians are in an intra-hospital network, the less the average cost of care at that hospital.

Locate the key physician Table 5 shows the top five most frequent specialties of nodes on referral paths and the top five cross-specialty referrals.

The RVU of a visit depends on the service performed or directly on the specialty of the physician. In the TDI dataset, the average RVU for physicians who specialize in cardiology, internal medicine, cardiac surgery and interventional cardiology are 4.00, 4.62, 3.65 and 3.69, respectively. The difference among specialties contributes to the uneven RVU sequence. We sort all physicians on a referral path to find the most key physician on it, allowing us to explore which kinds of physicians usually play the key role in treatment among all referral paths.

We define a new simple metric, according to which physicians on a referral path have the smallest aggregate RVU rank and PageRank centrality rank. For example, if a physician has the largest RVU among the physicians on a referral path, and the second largest PageRank value, the sum of rank would be three. Figure 4 shows several main groups of specialties often associated with the key physician, from which we find that physicians with specialties of cardiology, internal medicine and interventional cardiology occupy a relative central position in the national referral network.

Table 4 Percentage of change points in terms of increasing/decreasing trend in node position sequence of a referral path

\begin{tabular}{llllll}
\hline Year & 2007 & 2008 & 2009 & 2010 & 2011 \\
\hline Clustering coefficient & 75.0 & 74.9 & 74.9 & 74.8 & 74.7 \\
Betweenness centrality & 74.9 & 74.7 & 74.8 & 74.7 & 74.5 \\
Eigenvector centrality & 74.3 & 74.2 & 74.2 & 74.1 & 74.0 \\
PageRank centrality & 74.8 & 74.6 & 74.7 & 74.6 & 74.5 \\
h-index & 70.7 & 70.6 & 70.8 & 70.8 & 70.8 \\
\hline
\end{tabular}


Table 5 Referrals to physician specialties over 2006-2011

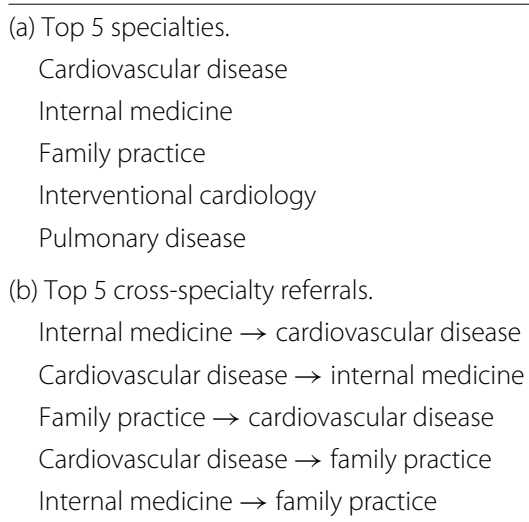

(b) Top 5 cross-specialty referrals.

Internal medicine $\rightarrow$ cardiovascular disease

Cardiovascular disease $\rightarrow$ internal medicine

Family practice $\rightarrow$ cardiovascular disease

Cardiovascular disease $\rightarrow$ family practice

Internal medicine $\rightarrow$ family practice

Node position in Referral Network vs. Feature of a node on Referral Paths Table 6 shows several strong correlations between node position measures (e.g., betweenness centrality) and referral path features. The strong correlations stem from the way we build referral networks with all referral paths. If more referral paths contain an edge, the nodes connected by the edge will have a more central position.

Preference of collaboration Sometimes a physician might have multiple options in terms of the target of a referral, especially when the physician is located in the center of referral networks with a wide range of connections. We compute the average number of common connected nodes for neighboring nodes in a referral path $P$, given by $\frac{\sum_{i=1}^{N-1}\left|V\left(P_{i}\right) \cap V\left(P_{i+1}\right)\right|}{N-1}$, where $V\left(P_{i}\right)$ is the set of neighboring nodes of node $P_{i}$ in the national referral network.

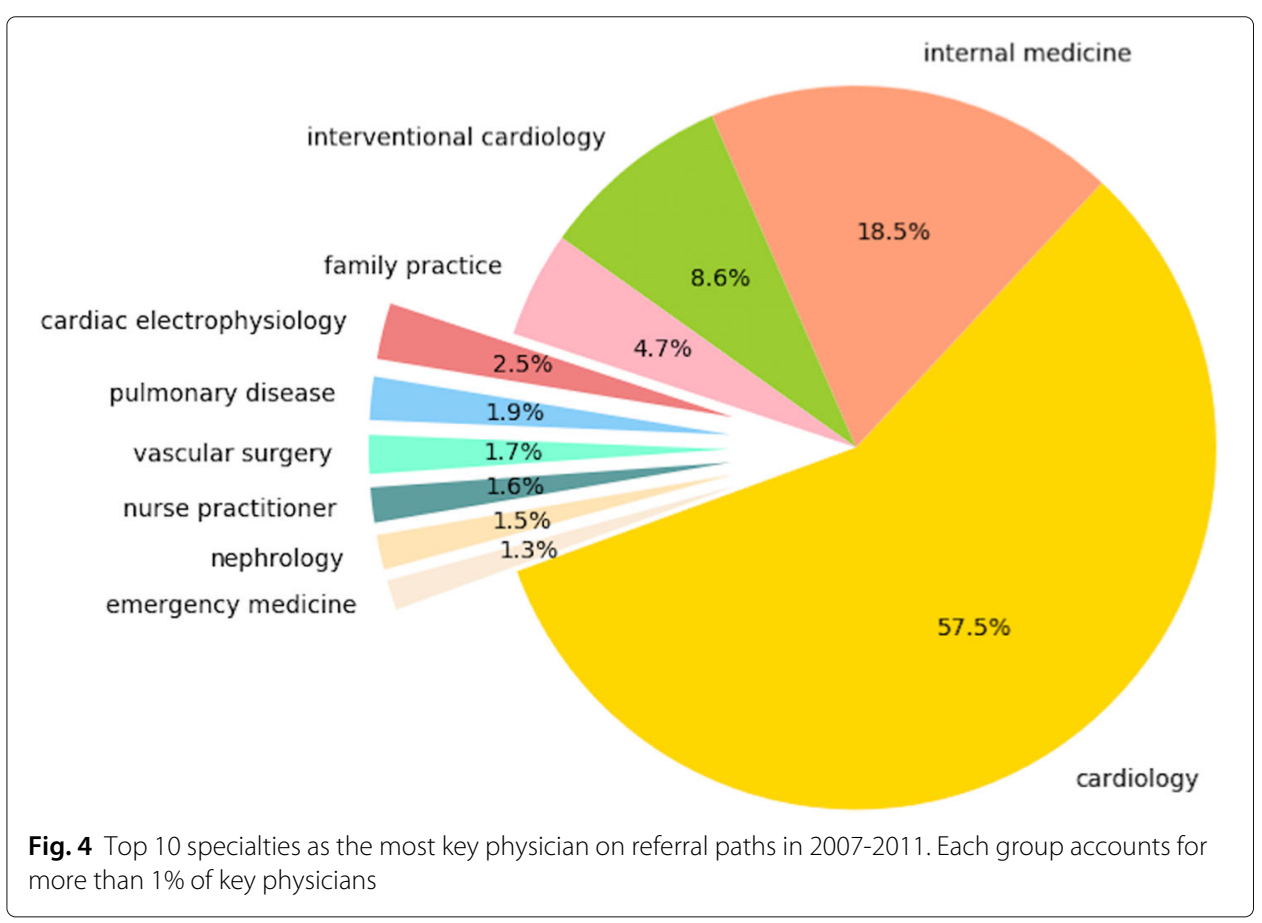


Table 6 Several pairs of strong correlations between node position and node feature on a referral path

\begin{tabular}{lll}
\hline Node position measure & Node feature about referral path & Correlation coefficient \\
\hline Betweenness centrality & \#paths with the node & 0.607 \\
PageRank centrality & \#paths with the node & 0.852 \\
PageRank centrality & \#paths with multiple occurrences & 0.740 \\
h-index & \#paths with the node & 0.783 \\
h-index & \#cross-PHN referral proposed by the physician & 0.640 \\
\hline
\end{tabular}

Table 7 shows that on average the neighbors or direct collaborators on a referral path have 25 common collaborators in the national referral network, while the expected number in a random network is $p(A X, B X \mid A B)=(N-2) \frac{(M-1)(M-2)}{\left(C_{2}^{N}-1\right)\left(C_{2}^{N}-2\right)}(N$ is the number of nodes, $M$ is the number of edges). Assume there is an edge between node $A$ and $B$. Then the remaining $N-2$ nodes are candidates for common neighbors. With $M-1$ edges remaining in the whole network and $C_{2}^{N}-1$ remaining pairs of possible edges, the probability that $A$ and a candidate neighbor $X$ are connected is $\frac{M-1}{C_{2}^{N}-1}$, which is almost the same as the ensuring conditional probability that $B$ and $X$ are connected. The sum of probabilities over $N-2$ candidates leads to the resulting probability being multiplied by $N-2$ to yield the expected value for the network. The clear gap in Table 7 supports a hypothesis that physicians tend to work with an acquaintance or someone in the same community when a referral is required. Among the referral steps of all referral paths in 2006-2011, only $33.2 \%$ are cross-PHN while $7.5 \%$ are cross-HRR referrals, which suggests that internal referral within the same hospital or HRR is the first choice. This suggests that actual geographic distance may be a factor for referral target selection. This would enable modeling of choice of referral targets as a ranking problem that would take into account geographic proximity (as well as possibly other factors).

\section{Three illustrative analyses}

\section{Teaching status classification}

About 220 U.S. hospitals are members of the Council of Teaching Hospitals and Health Systems (COTH) which provide professional resources to support clinical teaching environments. In this section we show how network-level and patient referral data can be used to validate their special status as well as to better articulate the features associated with membership of $\mathrm{COTH}$ as well as their implications for the functioning of these hospitals. We use the network features to classify whether a hospital is a COTH member based on hospital level (PHN) referral network features. This analysis is of particular interest from a health services perspective as there is a desire to identify hospitals that act as "health care hubs" or "referral hospitals"; the hospitals that patients are referred to when solutions to their medical needs are not found elsewhere. Such hospitals are likely to be key agents in the diffusion of new medical technologies and the exnovation of outdated technologies.

Table 7 Comparison of average common connected nodes between neighbors on a referral path and the expectation in a random network with the same size

\begin{tabular}{lllllll}
\hline Year & 2006 & 2007 & 2008 & 2009 & 2010 & 2011 \\
\hline Random network & $3.60 E-03$ & $3.10 E-03$ & $3.00 E-03$ & $2.90 E-03$ & $2.80 E-03$ & $2.80 E-03$ \\
Referral network & 25.13 & 24.64 & 24.95 & 24.97 & 24.95 & 24.96 \\
\hline
\end{tabular}


There is also a general interest in comparing the quality of services provided, patient outcomes, and costs of care between such referral hospitals and other hospitals. To date, a direct measure of a "referral hospital" does not exist and in lieu of that "teaching hospital" has been used as a surrogate. In this sub-section, we apply the notion of referral to posit direct measures of a referral hospital, evaluate the extent to which this correlates with COTH (Council of Teaching Hospitals) status, and evaluate the overall predictiveness of COTH status based on information in referral paths and the hospital network.

Physicians in a hospital might refer patients to other hospitals or receive patients from other hospitals. On a "traffic map", a node represents a hospital and an edge between two nodes represents the sum of cross-hospital referrals. The in- and out-degree of a hospital are the number of other hospitals with which a hospital sends or receives patients, respectively. An intuitively appealing measure of the extent to which a hospital is a problem-solver is given by the extent to which patients are referred to that hospital from other hospitals. That is, the more patients a hospitals receives as opposed to refers, the more that it might be thought of as a "referral hospital" in this sense. The difference in referrals received minus referrals sent from a hospital is termed "net patient flow" (NPF) and is given by

$$
N P F=\text { net patient flow }=\text { \#referrals in }- \text { \#referrals out. }
$$

We also compute the "net hospital degree" (NHD)

$$
N H D=\# \text { hospitals received patients from }- \text { \#hospitals send patients to }
$$

to capture the extent to which a hospital receives referrals from more hospitals than to which it sends patients.

In the health services literature (Desalegn 2013; Wu et al. 2010), COTH member hospitals have been considered referral or "go-to" hospitals. Therefore, we hypothesized that there should be a positive association between NPF/NHD and COTH status, and the higher the association, the more that the current health services definition of referral hospital is validated by these network definitions. To our knowledge, this is the first time correlates and predictors of COTH status have been examined.

NHD on the PHN traffic map alone cannot classify a hospital efficiently. In 2006-2011, of the group of hospitals with negative degree difference, $6.3 \%$ are teaching hospitals, while of the group of positive degree difference, $3.9 \%$ are teaching hospitals. Therefore, propensity to be a teaching hospital is strongly predicted by net (in minus out) degree, implying that the latter is a valid measure of the notion of a referral (or referral to hospital). We now incorporate more variables to assess if other features of the referral path or the network are associated with COTH status. Table 8 shows four groups of features of a hospital (PHN).

Because we have a binary classification problem on a middle-size dataset (about 4800 hospitals), we apply the following models: logistic regression (LR), K-nearest neighbors (KNN), support vector machine (SVM), decision tree (DT), random forest (RF), gradient boosting decision tree (GBDT), AdaBoost with decision trees and an equal-weighted voting method based on all the models. To reduce the risk of reverse-causality, the predictors are measured piror to the COTH label being measured, which was post 2011.

Since more than $95 \%$ of the 4800 hospitals are not teaching hospitals, only a limited number of predictors can be included in the model as there is less information with which 
Table 8 The feature list of a hospital (PHN level referral network) for teaching hospital classification

\begin{tabular}{ll}
\hline Feature Group & Features \\
\hline PHN level network measures & \#nodes, \#edges, gini coefficient of indegree distribu- \\
& tion, gini coefficient of outdegree distribution, alpha \\
& of indegree power law test, alpha of outdegree power \\
& law test, diameter, global clustering coefficient, local \\
& clustering coefficient, (in, in) assortativity, self degree \\
correlation, reciprocity of \# referral, reciprocity of RVUs
\end{tabular}

Difference (in - out) of edge weights on PHN traffic map

PHN position on PHN traffic map

average feature of referral paths in the PHN

Average node position of the PHN in the national referral network
Degree, \#different referred patients, \#referral, geometric mean of \#visit, geometric mean of RVUs, ranking index based weight

Local clustering coefficient, PageRank, h-index

Length, avg-time-gap, avg-time-range, recurrent node, \# nodes before recurrence, phy-entropy, PHNentropy, HRR-entropy, common connected nodes between neighbors, bidirectional pairs

Local clustering coefficient, PageRank, h-index

to build the classification model than if $50 \%$ of the hospitals were not teaching hospitals. Define negative (" 0 ") as non-teaching status and positive (" 1 ") as teaching status, $t p$ is true positive, $f p$ is false positive, $f n$ is false negative. Then we have three measures based on the confusion matrix of the accuracy of the predictions: $\operatorname{precision}(p)=\frac{t p}{t p+f p} \quad \operatorname{recall}(r)=$ $\frac{t p}{t p+f n} \quad$ F-score $=\frac{2 p r}{p+r}$, the harmonic mean.

To obtain a maximally interpretable model, the non-significant predictors in the above LR model and one pair of any highly collinear predictors were removed one at a time until no more predictors could be ruled out. This same procedure was applied to the interpretative versions of the predictive models of treatment and outcome following treatment and is presented later in the paper.

Table 9 shows the best two models according to the overall F-score metric. Table 10 shows some significant predictors in the Logistic Regression (LR) model with their estimated value and confidence intervals. They support the idea that referral path and referral network features matter for COTH label classification. If a feature has a positive coefficient, it means that an increase in the feature tends to make the model predict the hospital as a teaching hospital. For example, the more that referrals into the hospital exceed those departing from the hospital, the greater the NPF and the likelihood that the hospital is a COTH. Likewise, hospitals with a high h-index and long-time referral paths are more likely to be COTH hospitals. These findings make intuitive sense as it is reasonable to expect that the most complex clinical cases will on average generate the longest referral

Table 9 COTH classification results of Logistic Regression (LR) and Support Vector Machine (SVM)

\begin{tabular}{llllllll}
\hline LR & 2006 & 2007 & 2008 & 2009 & 2010 & 2011 & average F-score \\
\hline Recall & 0.844 & 0.902 & 0.805 & 0.882 & 0.830 & 0.792 & \\
Precision & 0.704 & 0.712 & 0.733 & 0.667 & 0.780 & 0.792 & \\
F-score & 0.768 & 0.796 & 0.767 & 0.759 & 0.804 & 0.792 & 0.781 \\
SVM & & & & & & & \\
Recall & 0.791 & 0.762 & 0.717 & 0.774 & 0.825 & 0.914 & \\
Precision & 0.756 & 0.780 & 0.805 & 0.750 & 0.750 & 0.762 & \\
F-score & 0.773 & 0.771 & 0.759 & 0.762 & 0.786 & 0.831 & 0.780 \\
\hline
\end{tabular}

They are the best two models in terms of average F-score in 2006-2011 
Table 10 Significant predictors in Logistic Regression for COTH classification

\begin{tabular}{|c|c|c|c|}
\hline Feature Name & Estimated Coefficient & 95\% Confidence Interval & $P$-value \\
\hline $\begin{array}{l}\text { Gini coefficient of degree distribu- } \\
\text { tion in PHN network }\end{array}$ & 2.823 & $(0.8444 .802)$ & $5.18 \mathrm{E}-03$ \\
\hline $\begin{array}{l}\text { Global clustering coefficient of PHN } \\
\text { network }\end{array}$ & -10.693 & $(-13.218-8.167)$ & $<2 \mathrm{E}-16$ \\
\hline (in, in) degree assortativity & 4.813 & $(2.9816 .646)$ & 2.63E-07 \\
\hline $\begin{array}{l}\text { Difference (in-out) of \# referrals on } \\
\text { PHN traffic map }\end{array}$ & 3.678 & (1.630 5.726) & $4.32 \mathrm{E}-04$ \\
\hline $\begin{array}{l}\text { h-index of a hospital on the PHN } \\
\text { traffic map }\end{array}$ & 7.862 & $(5.8779 .847)$ & $8.37 \mathrm{E}-15$ \\
\hline Avg time range of a referral path & 5.138 & $(2.1578 .119)$ & 7.29E-04 \\
\hline $\begin{array}{l}\text { ratio of referral paths with recurrent } \\
\text { nodes }\end{array}$ & -12.950 & $(-16.614-9.286)$ & 4.29E-12 \\
\hline Avg \#nodes before recurrent nodes & 6.139 & $(3.8448 .434)$ & $1.58 \mathrm{E}-07$ \\
\hline $\begin{array}{l}\text { Avg \#bidirected pairs on referral } \\
\text { paths in the PHN }\end{array}$ & 6.459 & $(2.407$ 10.512) & $1.78 \mathrm{E}-03$ \\
\hline
\end{tabular}

paths. However, an in-depth study that looks at medical detail beyond that captured in claims data will be needed to validate that the most severe and complex medical cases do generate the longest referral paths as opposed to an alternative explanation such as health care inefficiency.

\section{Patient clinical outcome and treatment received classification}

We next explore whether it is possible to predict the treatment outcome for a patient based on the measures and features of the physician referral network and the referral path. Here we take a dataset of Medicare patients diagnosed with Acute Myocardial Infarction (AMI) over 2006-2011, which by virtue of the serious nature of the medical event was always diagnosed in a hospital setting. Because AMI embodies a small subset of the total claims with cardiovascular disease diagnoses, these claims are a small subset of the claims used to construct the data set of referral paths and the associated physician network. Therefore, there is no tautological dependency between the referral-path and network-based predictors based on the ensemble of cardiovascular care and the treatment outcomes of patients who experienced an AMI. The Medicare claims data record is analyzed for each patient to determine the treatments the patient received post-diagnosis and key follow-up medical events. The dataset has the following key attributes: Bene ID, admission date, death1yr (death or not within one year after index admitted date), PCI (indicator of Percutaneous Coronary Intervention within one year after index admitted date), total_payment_1yr (total real payment within one year after index admitted date). By matching the AMI admission date with the date of visit to the first physician on a referral path for the same beneficiary, we get more than 100,000 pairs of referral paths and the corresponding AMI treatment and outcome variables. If we relax the gap between the AMI admission date and the first referral path visit date to one day (as opposed to being an exact 0-day match), there will be about 22,000 more records. A further relaxation to two-days yields 3800 more records. To be cautious, we use the 0 -day matching rule in the following.

The outcome death1yr and treatment PCI are both binary-valued random variables. We collect 69 kinds of features in Table 11 from referral path and patient referral network analysis, which are in six groups: network measures of the dominant HRR on the referral 
Table 11 Feature list of a referral path for treatment outcome classification/regression

\begin{tabular}{ll}
\hline Group of Features & Features and ID \\
\hline Network measures in the dominant HRR & 1:\#nodes, 2:\#edges, 3:indegree gini coefficient, 4:outdegree \\
& gini coefficient, 5:indegree power law test alpha, 6:outdegree \\
& power law test alpha, 7: diameter, 8:global clustering coef- \\
& ficient, 9:local clustering coefficient, 10: (in, in) assortativity, \\
& $11:$ self in/out degree coefficient, 12:referral reciprocity, 13:RVU \\
& reciprocity
\end{tabular}

Referral path sequence

Average node positions on the referral path

Average weights of edges in the national referral network covered by the referral path Last physician on the referral path

Patient history information 14:\#nodes, 15:average time gap, 16: time range, 17:indicator of recurrence, 18: \#nodes before recurrence, 19:physician distribution entropy, 20: PHN distribution entropy, 21:HRR distribution entropy, 22:average \#common connected nodes between neighbors, 23:\#pairs of nodes with reciprocal referrals, 37:\#change points, 38:\#previous referral path in the same year, 39: distance between the first visited hospital and the end one, 40:total RVU, 41:month of the first visit, 42:\#visited teaching hospitals, 43:specialty of the key physician, 44:specialty of the last physician, 45:\#visited PHN with negative (in-out) degree on PHN traffic map, 46:\#visited PHN with positive (in-out) degree on PHN traffic map, 47:sum of (in-out) degree for all PHN on the referral path, 60:indicator of admitted by emergency department for the first node

24:local clustering coefficient, 25:PageRank, 26:h-index, 27:\#paths which contains the node, 28:\#paths where the node is the starting one, 29:\#paths where the node is the end one, 30:index of the first-time occurrence, 31:\#paths where the node occurs multiple times, 32:\#cross-HRR referrals proposed by the node, 33:\#cross-PHN referrals proposed by the node

34:\#referrals, 35:RVU, 36:ranking based weight

48:RVU, 49:month of visit, 50:local clustering coefficient, 51:PageRank, 52:h-index, 53:\#paths which contains the node, 54:\#paths where the node is the starting one, 55 :\#paths where the node is the end one, 56:average index of the first-time occurrence, 57:\#paths where the node occurs multiple times, 58:\#cross-HRR referrals proposed by the node, 59:\#cross-PHN referrals proposed by the node

61:age, 62:indicator of HIV, 63:indicator of asthmatic lung disease, 64:indicator of cancer, 65:indicator of dementia, 66:indicator of diabetes, 67:indicator of liver disease, 68:indicator of chronic non-asthmatic lung disease, 69:indicator of chronic renal disease

path, referral path features (e.g., number of nodes, time range), average node positions on the referral path, average weights of edges in the national referral network covered by the referral path, features of the last physician on the referral path (e.g., PageRank value, \#cross-PHN referral proposed by the physician), basic patient information (e.g., age).

In addition to the seven traditional classification models (LR, KNN, SVM, DT, RF, GBDT, AdaBoost), we try to boost the performance of classification with the following methods.

- Feature engineering. Encoding categorical attributes, such as specialty of the key physician and the month of admission date. Features are extracted using both the exact matching referral path with the AMI record and the immediately preceding referral path within the 90 day period before the exact matching one, in order to capture the association between referral path features and subsequent treatment outcomes.

- 10-fold cross validations. Accomplished by partitioning the original sample into a training set and a test set in rotation. 
- Undersampling. Undersample some training cases to balance the ratio of positive/negative in training set.

- Feature selection. Apply Random Forest (RF) to sort features by their importance (Genuer et al. 2010), and pick up a subset of important features for classification models. Here the importance of a given feature is the increase in mean error of a tree in the forest when the observed values of this feature are randomly permuted.

- Voting for the final label. Collect prediction result of each classification model and vote for the final prediction result of a test case.

- Xgboost (Chen and Guestrin 2016). Upgrade the gradient boosting model from GBDT to Xgboost, which aims to strengthen regularization of trees and control overfitting.

GBDT has the highest F-score with its performance depicted in Table 12 for each year and outcome. Since we can tune parameters in a classification model to get a higher recall or precision, the F-score is more meaningful as an overall evaluation metric. The moderate F-score suggests that a lot of unmeasured variables contribute to treatment decisions and patients' survival. The lack of clinical detail and personal information such as heart rate and blood pressure weakens the power of machine learning models, but the referral path features and network measures support the above models to beat random prediction while the accuracy is almost as good as that of other diagnosis classification. A complex convolutional neural network (CNN) model (Fiterau et al. 2017) aims to predict osteoarthritis with much more (600+) directly related features (e.g., clinical measures, joint symptoms/function) and 7-day time series accelerometer sensory data, but the accuracy of baselines and the $\mathrm{CNN}$ ranges from 0.633 to 0.789 . Table 13 shows the average F-score for death1yr and PCI classification on two separate groups divided by age. The power of referral path features differs, which means age is an important factor. As predictability does not necessarily imply causality, to attain rigorous causal inferences to the standard typical in medical research would require more study regarding potential confounding variables and possibly involve a randomized study. Moreover, if available, we should group by referral paths based on clinical tests and demographics, because it will be clear to see the effects of referral paths among a group of similar patients before treatment.

Table 14 shows the top 10 important features for two indicators in 2011, which are selected by the result of RF (Genuer et al. 2010). For both death1yr and PCI, average time gap on the referral path is one of the most important features. We conjecture that the gap reflects whether the case is serious. In addition, total RVU of physicians on the referral path is predictive of death1yr (the patient outcome) and physician position (measured by

Table 12 Classification results of GBDT for death1yr and PCI in 2007-2011

\begin{tabular}{lllllll}
\hline PCl & 2007 & 2008 & 2009 & 2010 & 2011 & Average F-score \\
\hline Recall & 0.703 & 0.700 & 0.702 & 0.695 & 0.694 & \\
Precision & 0.572 & 0.574 & 0.585 & 0.597 & 0.607 & \\
F-score & 0.631 & 0.630 & 0.638 & 0.642 & 0.647 & 0.638 \\
death1yr & & & & & & \\
Recall & 0.702 & 0.698 & 0.710 & 0.704 & 0.682 & \\
Precision & 0.640 & 0.632 & 0.639 & 0.650 & 0.633 & \\
F-score & 0.669 & 0.663 & 0.672 & 0.675 & 0.657 & 0.667 \\
\hline
\end{tabular}


Table 13 Average F-score in 2007-2011 of GBDT on groups divided by age

\begin{tabular}{lll}
\hline & Death1yr & $\mathrm{PCl}$ \\
\hline Age $<=75$ & 0.592 & 0.695 \\
Age $>75$ & 0.687 & 0.565 \\
\hline
\end{tabular}

PageRank) is predictive of PCI (the patient treatment received). Moreover, Table 15 contains some significant predictors in the logistic regression (LR) model for the two binary indicators. The above significant features offer new directions for medical researchers to investigate with their domain knowledge.

GBDT's level of predictive accuracy was on average higher than LR for predicting PCI and higher than LR for predicting death within a year. However, the form of the model from LR is the most amenable to interpreting the model and determining which terms are the most predictive. For this reason, Table 15 shows the estimated parameter values, 95\% confidence interval limits, and $p$-values for the PCI treatment selection and the 1year death models. The direction of the estimated coefficients might be helpful to reveal some important relationships. For example, for Feature 34 (weights of edges covered by the referral path) in the dealth1yr model, the estimated coefficient implies that referrals of a patient to core physicians in the referral network are associated with death within the year. For Feature 25 (average PageRank value of physicians on a referral path) in the PCI model, the positive coefficient suggests that core physicians tend to treat the patients who are most likely to undergo PCI.

\section{Linear regression analysis of log(total $1 \mathrm{yr}$ payments)}

In addition to categorical treatment outcome variables, we also use regression to explore the relationship between referral path features and total_payment_1yr. A feature of Medicare claims data is that any patient in the dataset must have had at least one encounter with a physician in order to enter the dataset. Therefore, their annual cost of care will be non-zero. However, cost data is notorious for exhibiting right skew. Therefore, we model $\log ($ Total Cost) as opposed to Total Cost itself using a linear regression model.

Table 14 Top 10 important features for death1yr and PCl generated by Random Forest feature selection method (Genuer et al. 2010)

\begin{tabular}{|c|c|c|}
\hline Rank & Death1yr & $\mathrm{PCl}$ \\
\hline 1 & Total RVU of the referral path & Average time gap on the referral path \\
\hline 2 & Total RVU of the previous referral path & Indicator of patient's age in 66-70 \\
\hline 3 & Average time gap on the referral path & $\begin{array}{l}\text { Average PageRank values of all physicians on the } \\
\text { referral path }\end{array}$ \\
\hline 4 & Time range of the referral path & $\begin{array}{l}\text { Indicator of the key physician's specialty on the } \\
\text { referral path as "interventional cardiology" }\end{array}$ \\
\hline 5 & $\begin{array}{l}\text { Average index of the first-time occurrence on a } \\
\text { referral path for the last physician }\end{array}$ & Indicator of patient's age in 76+ \\
\hline 6 & $\begin{array}{l}\text { Local clustering coefficient of the last physician } \\
\text { on the referral path }\end{array}$ & $\begin{array}{l}\text { The number of referral paths that include the last } \\
\text { physician }\end{array}$ \\
\hline 7 & $\begin{array}{l}\text { Times of being the end node on a referral path of } \\
\text { the last physician on the referral path }\end{array}$ & $\begin{array}{l}\text { Indicator of the key physician's specialty on the } \\
\text { referral path as "interventional cardiology" }\end{array}$ \\
\hline 8 & $\begin{array}{l}\text { Times of being the first node on a referral path for } \\
\text { the last physician }\end{array}$ & $\begin{array}{l}\text { Average \#involved paths among physicians on } \\
\text { the referral path }\end{array}$ \\
\hline 9 & indicator of patient's age in 76+ & $\begin{array}{l}\text { Average times of being the first node on a referral } \\
\text { path for all physicians on the referral path }\end{array}$ \\
\hline 10 & $\begin{array}{l}\text { Average times of being the end node on a referral } \\
\text { path for all physicians on the referral path }\end{array}$ & $\begin{array}{l}\text { Times of being the first node on a referral path for } \\
\text { the last physician }\end{array}$ \\
\hline
\end{tabular}


Table 15 Significant predictors for LR on two binary treatment variables with estimated coefficients in $95 \%$ confidence interval $(\mathrm{Cl})$

\begin{tabular}{|c|c|c|c|}
\hline \multicolumn{4}{|l|}{ (a) death1yr } \\
\hline Feature & Estimate & $95 \% \mathrm{Cl}$ & $p$-value \\
\hline \#nodes in domain HRR & -0.243 & $(-0.389-0.098)$ & $1.03 E-03$ \\
\hline Physician distribution entropy & -0.313 & $(-0.625-0.0013)$ & 0.049 \\
\hline PHN distribution entropy & -0.528 & $(-0.692-0.365)$ & $2.34 E-10$ \\
\hline \#pairs of nodes with reciprocal referrals & -2.496 & $(-3.666-1.325)$ & $2.93 E-05$ \\
\hline Avg. PageRank values on a referral path & -2.290 & $(-2.803-1.778)$ & $<2 E-16$ \\
\hline Avg. index of first occurrence & -0.569 & $(-0.974-0.164)$ & 0.0059 \\
\hline Avg. proposed \#cross-PHN referrals & 1.628 & $(0.9612 .295)$ & $1.73 E-06$ \\
\hline Avg. \#referrals on the corresponding edges & 8.696 & $(4.77112 .620)$ & $1.41 E-05$ \\
\hline Avg. ranking-based weight on the corresponding edges & -3.973 & $(-6.426-1.519)$ & 0.0015 \\
\hline \#previous paths & 2.204 & (1.908 2.500) & $<2 E-16$ \\
\hline Total RVU & 11.414 & (10.461 12.367) & $<2 E-16$ \\
\hline Times of being the end node of the last physician & -2.985 & $(-4.075-1.896)$ & $7.89 E-08$ \\
\hline Avg. first occurrence index of the last physician & 4.869 & $(4.1765 .562)$ & $<2 E-16$ \\
\hline $\begin{array}{l}\text { Times of occurring multiple times of the last physician } \\
\text { (b) } \mathrm{PCl}\end{array}$ & 1.778 & $(1.0412 .514)$ & $2.23 E-06$ \\
\hline Feature & Estimate & $95 \% \mathrm{Cl}$ & $p$-value \\
\hline Physician distribution entropy & -0.368 & $(-0.678-0.058)$ & 0.019 \\
\hline PHN distribution entropy & 0.547 & $(0.3590 .734)$ & $1.08 E-08$ \\
\hline Avg. \#common connected nodes between neighbors & 0.487 & $(0.0970 .877)$ & 0.014 \\
\hline Avg. PageRank values on a referral path & 3.874 & $(3.3374 .411)$ & $<2 E-16$ \\
\hline Avg. proposed \#cross-PHN referrals & -1.738 & $(-2.278-1.197)$ & $2.89 E-10$ \\
\hline Avg. \#referrals on the corresponding edges & -2.222 & $(-3.822-0.622)$ & 0.0065 \\
\hline \#previous paths & -1.845 & $(-2.155-1.533)$ & $<2 E-16$ \\
\hline Total RVU & -2.113 & $(-2.909-1.315)$ & $2.02 E-07$ \\
\hline Local clustering coefficient of the last physician & -1.352 & $(-1.969-0.735)$ & $1.76 E-05$ \\
\hline Avg. first occurrence index of the last physician & -3.024 & $(-4.034-2.013)$ & $4.48 E-09$ \\
\hline
\end{tabular}

$$
\log \left(Y_{t}\right)=\lambda_{t}+\boldsymbol{\beta}_{1}^{T} \boldsymbol{X}_{t}+\boldsymbol{\beta}_{2}^{T} \boldsymbol{X}_{t} t+\varepsilon_{t}
$$

The form of the model is given in Eq. 4, where $Y_{t}$ and $X_{t}$ are dependent treatment outcome variables (e.g., total 1yr payments) and the vector of referral path features, respectively, with the outcomes measured over 2007-2011. The network related measures in $X_{t}$, which are only measured once per calendar year, are lagged in the years of 2006-2010 to make sure that $Y_{t}$ is measured after $X_{t}$.

The main effect of the vector of referral path features is $\beta_{1}$ while its modification by year $t$ is $\beta_{2}$, although in our primary analysis we focus on the model in which $\beta_{2}=0$. The parameters $\lambda_{t}$ allow for an unstructured trend across time, and $\varepsilon_{t} \sim N\left(0, \sigma_{\log (y)}^{2}\right)$ describes the distribution of the error term. Modeling time in 2007-2011 categorically as a main effect and linearly as a modifier of referral path features serves the purpose of allowing a maximally flexible trend.

The fitted models appear to have a high level of face validity. For example, higher RVU is associated with greater total cost of treatment. Other significant predictors of $\log$ (total_payment_1yr), their estimated coefficients $\boldsymbol{\beta}_{1}, 95 \%$ confidence intervals, and $p$ values are also shown in Table 16. The asterisk represents significant interactions with time, which is assessed by estimating the full model in (4). 
Table 16 Significant predictors in multiple linear regression models for log(Total 1yr payments) with estimated coefficients in \%95 confidence interval (Cl)

\begin{tabular}{llll}
\hline Feature & Estimate & Cl & $p$-value \\
\hline \#nodes in domain HRR & 0.121 & $(0.0990 .142)$ & $<2 E-16$ \\
referral reciprocity in domain HRR & 0.209 & $(0.1670 .251)$ & $<2 E-16$ \\
\#nodes* & -2.588 & $(-2.992-2.183)$ & $<2 E-16$ \\
Physician distribution entropy & 1.365 & $(1.3211 .410)$ & $<2 E-16$ \\
PHN distribution entropy* & 0.413 & $(0.3470 .480)$ & $<2 E-16$ \\
Avg. \#common connected nodes between neighbors & -0.357 & $(-0.432-0.282)$ & $<2 E-16$ \\
\#pairs of nodes with reciprocal referrals & 2.618 & $(2.3742 .863)$ & $<2 E-16$ \\
Avg. local clustering coefficient on the referral path & -1.222 & $(-1.326-1.117)$ & $<2 E-16$ \\
Avg. PageRank values on the referral path & 0.983 & $(0.8881 .077)$ & $<2 E-16$ \\
Avg. index of first occurrence on the referral path & 0.341 & $(0.2350 .447)$ & $3.05 E-10$ \\
Avg. proposed \#cross-PHN referrals & -0.592 & $(-0.685-0.498)$ & $<2 E-16$ \\
Avg. \#referrals on the corresponding edges & -0.567 & $(-0.902-0.232)$ & $9.25 E-04$ \\
Avg. ranking-based weight on the corresponding edges* & 0.775 & $(0.4851 .064)$ & $1.59 E-07$ \\
\#previous paths* & 0.304 & $(0.2120 .396)$ & $9.28 E-11$ \\
Total RVU* & 5.028 & $(4.6045 .451)$ & $<2 E-16$ \\
Month of the first visit & categorical & vary for groups & $<2 E-16$ \\
Specialty of the key physician & categorical & vary for groups & $<2 E-16$ \\
Month of the last visit & categorical & vary for groups & $<2 E-16$ \\
Avg. first occurrence index of the last physician* & -0.433 & $(-0.686-0.179)$ & $7.99 E-04$ \\
\hline Astersk m & & &
\end{tabular}

Asterisk means the predictor has significant interactions with time

\section{In-depth study of a hospital}

Figure 5 shows a PHN level patient referral network. Table 17 shows the weights of some directed edges. The hospital is a non-teaching hospital, the Mark Twain Medical Center (in CA), with national provider ID 051332. The $p$-values of the indegree and outdegree distribution power law test are 0.21 and 0.73 , respectively. The global clustering coefficient is 0.37 and the local clustering is 0.35 . The average PageRank value of all nodes in

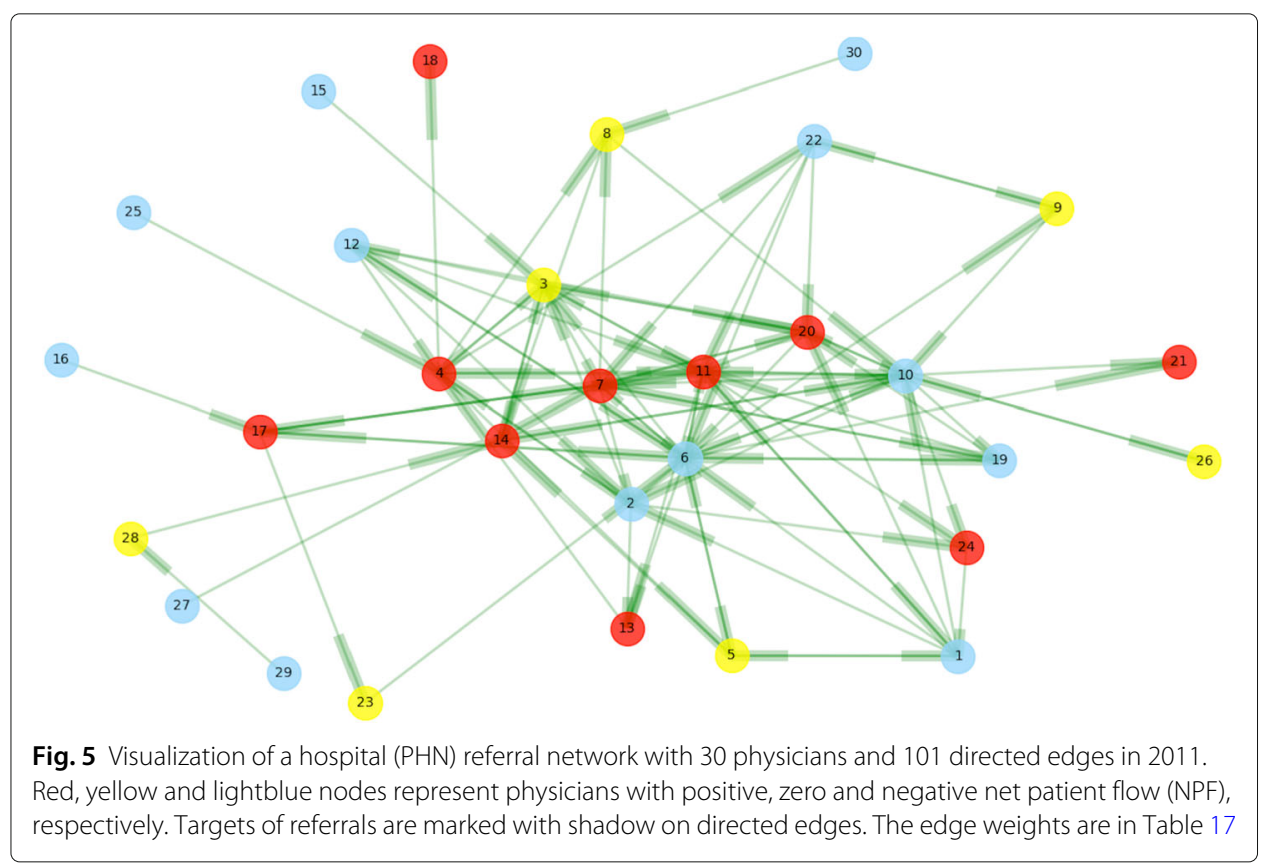


Table 17 A part of network weights in the PHN network of Fig. 5

\begin{tabular}{lllll}
\hline$(s, t, w)$ & $(s, t, w)$ & $(s, t, w)$ & $(s, t, w)$ & $(s, t, w)$ \\
$(20,7,2)$ & $(1,6,2)$ & $(22,6,2)$ & $(6,7,11)$ & $(10,7,2)$ \\
$(7,6,10)$ & $(6,10,3)$ & $(7,19,2)$ & $(3,12,2)$ & $(12,2,2)$ \\
$(17,6,2)$ & $(17,11,3)$ & $(6,11,4)$ & $(7,17,3)$ & $(14,3,2)$ \\
$(6,17,5)$ & $(8,10,2)$ & $(14,10,2)$ & $(19,6,3)$ & $(4,2,2)$ \\
$(2,4,4)$ & $(10,11,3)$ & $(11,24,2)$ & $(3,14,2)$ & $(11,6,2)$ \\
\hline
\end{tabular}

The weights (i.e. number of referrals) of the remaining edges are one. A triple means (source, target, weight)

the national referral network is $1.71 \times 10^{-6}$. On the hospital traffic map, the net hospital degree (NHD) is 3 and the NPF is -43 .

Some nodes have more connections with others in the hospital (PHN), such as physician 6. The PageRank value of physician 6 is $5.36 \times 10^{-6}$ while that of the physician 15 , who is on the periphery, is $2.07 \times 10^{-6}$. In the 2011 national referral network, physician 6 is the first/end node in a referral path 38/26 times among 75 occurrences in total. The average index of the first occurrence in a referral path is 2.03. Physician 6 initiates 24 cross-HRR referrals and 49 cross-PHN referrals.

The following are some overall features based on all referral paths whose dominant HRR is the hospital. The average length is 3.10, average time range is 25.45 (days), $30 \%$ of referral paths have recurrent nodes, each referral path has 0.37 pairs of bidirected nodes, 3.67 nodes are before the recurrent nodes, entropy of physician distribution is 1.257 , entropy of hospital distribution is 0.71 , entropy of HRR distribution is 0.55 , the neighboring nodes on referral paths have 7.2 common connected neighbors in the national referral network. The above referral path-related features are applied to predict the COTH label of the hospital.

The following is an example of a referral path: a patient visited Node 19, Node 6, an external Node E1 (some node in another hospital), Node 6, Node E1, Node 17 within 45 days. For the neighboring nodes on the referral path, on average they had 7.8 nodes (physicians) in common connection in 2011. The total RVU during the period of referrals was 20.52. No teaching hospital provided treatment to the patient. Four of the six physicians worked in a hospital (PHN) with negative NHD. Among 31 PHNs which had connections with the PHN 051332 in Fig. 5, PHN 050084 St. Joseph's Medical Center in CA, sent and received the most patients to and from PHN 051332. The straight-line distance between the two hospitals is only $59 \mathrm{~km}$. Since they are located in the vast rural area of CA, their relationship reflects the "gravity law" described in (An et al. 2018).

\section{Conclusions}

In this paper, we apply algorithms and models from network science, statistics and machine learning to define the notion of the referral path and to derive features of interest to explore patterns in the U.S.-based cardiovascular patient referral networks. Firstly, since referral path features mainly describe micro-patterns related to patient referrals, a better understanding of referral features may provide insights into directions for improving the healthcare system. For example, node position values on a referral path change frequently in terms of increasing/decreasing trend, so we realize the significant role of physicians with relative larger centrality measures in referral networks when they build connections in the network. Physicians could also identify their position in the large community of physicians and set a goal of collaborations for career development. 
Moreover, physicians tend to send a patient to another physician who has a lot of common connected neighbors in the national referral network, suggesting that network structural position of physicians is a marker of their reputation and prominence among their colleagues. Our pattern-mining processing of the millions of cardiovascular disease treatment records and subsequent network, statistical and big data analysis can readily be applied to other diseases. Ultimately, we hope to develop a network science toolkit (measures, tools, and models) available to medical researchers for use in their own research.

Second, we explored applications of the referral paths features and referral network measures on teaching hospital (COTH) classification for 4,800 hospitals in the U.S. The fact that the referral-path definitions of a "referral" or "hub" hospital based on differences of patient flows into and from a hospital were strong predictors but far from perfect predictors of COTH status is encouraging vis-a-vis the utility of these new measures. On the one hand, it provides a form of face validity of the referral-path based measures; if they had no predictive power we would doubt their validity. However, by being far from perfect predictors of COTH status, this leaves open the strong possibility that a much more informative measure or indicator of a referral (problem-solving) hospital can be constructed. Such a measure will aid health services researchers and other researchers interested in the structure and consequences of the U.S. healthcare system. We believe hospitals could learn from the patterns and results derived herein in the following ways: when a successful new treatment is approved, identifying the network positions or features of the first physicians and hospitals to adopt may help to enable such influential physicians to be identified as well as provide network-based insights into the keys underlying their success. Those non-teaching hospitals that are most like teaching hospitals in their network characteristics might be the best candidates to become teaching hospitals. Analogously, those teaching hospitals that are the most like non-teaching hospitals might be in need to re-structuring.

Third, by linking AMI treatment and outcome variables to the corresponding referral paths, we find several informative predictors with either larger feature importance or significant effects, such as the time gap between two visits on the referral path and the total RVUs of all physicians' endeavors. The novelty of these referral path measures suggests that a deeper look into their significance is warranted. We have only just scratched the surface of the enormous potential for using referral path features to improve predictions of treatment received and treatment outcomes. Understanding referral path patterns has the potential to ultimately help hospitals, physicians and patients towards the ultimate goal of building an optimal referral path for each patient with a better treatment outcome and providing the most effective allocation of medical resources.

\section{Endnotes}

${ }^{1}$ RVU stands for "relative value unit". This is a Medicare invention used in the calculation of reimbursements that encodes the "value" of a given procedure.

${ }^{2}$ The Dartmouth Institute for Health Policy and Clinical Practice

${ }^{3}$ Example fields include city, HRR, state, zip code and teaching type of the hospital, the specialty of the physician, etc.

${ }^{4}$ The list of positions - denoting first, second,...,nth - in the sequence of $n$ visits that make up the referral path. 


\begin{abstract}
Abbreviations
AMI: Acute myocardial infarction; Cl: Confidence interval; CNN: Convolutional neural network; COTH: Council of teaching hospitals and health systems; DT: Decision tree; GBDT: Gradient boosting decision tree; HRR(s): Hospital referral region(s); KNN: K-nearest neighbors; LR: Logistic regression; NHD: Net hospital degree; NPF: Net patient flow; NPI: National provider identification; PCI: Percutaneous coronary intervention; PHN: Referral network in hospital level; RF: Random forest; RVU: Relative value unit; SVM: Support vector machine; TDI: The dartmouth institute for health policy and clinical practice
\end{abstract}

\title{
Acknowledgements
}

The authors thank Jonathan S. Skinner and Julie P.W. Bynum at Geisel School of Medicine of Dartmouth College for help in obtaining the data.

\section{Funding}

Research for the paper was supported by NIH grants U01 AG046830 and P01 AG019783.

\section{Availability of data and materials}

The datasets in this paper are authorized by The Dartmouth Institute for Health Policy and Clinical Practice (TDI), under the privacy policy of Protected Health Information (PHI).

\section{Authors' contributions}

All authors discussed the features \& models, edited the manuscript. CA implemented the analysis. All authors read and approved the final manuscript.

\section{Competing interests}

The authors declare that they have no competing interests.

\section{Publisher's Note}

Springer Nature remains neutral with regard to jurisdictional claims in published maps and institutional affiliations.

\section{Author details}

${ }^{1}$ Department of Computer Science, Dartmouth College, 03755 Hanover, NH, USA. ${ }^{2}$ Department of Biomedical Data Science and the Dartmouth Institute of Health Policy and Clinical Practice in the Geisel School of Medicine at Dartmouth College, 03784 Lebanon, NH, USA. ${ }^{3}$ Department of Mathematics, Dartmouth College, 03755 Hanover, NH, USA. ${ }^{4}$ External Faculty, The Santa Fe Institute, Santa Fe, NM 87501, USA.

\section{Received: 3 April 2018 Accepted: 11 July 2018}

Published online: 31 July 2018

\section{References}

An C, O'Malley AJ, Rockmore DN, Stock CD (2018) Analysis of the US patient referral network. Stat Med 37(5):847-866

Ball TM, Stein MB, Ramsawh HJ, Campbell-Sills L, Paulus MP (2014) Single-subject anxiety treatment outcome prediction using functional neuroimaging. Neuropsychopharmacology 39(5):1254

Barnett ML, Christakis NA, O'Malley AJ, Onnela J-P, Keating NL, Landon BE (2012) Physician patient-sharing networks and the cost and intensity of care in us hospitals. Med Care 50(2):152

Burns LR, Muller RW (2008) Hospital-physician collaboration: Landscape of economic integration and impact on clinical integration. Milbank Q 86(3):375-434

Bynum JP, Bernal-Delgado E, Gottlieb D, Fisher E (2007) Assigning ambulatory patients and their physicians to hospitals: A method for obtaining population-based provider performance measurements. Health Serv Res 42(1 p1):45-62

Chen T, Guestrin C (2016) Xgboost: A scalable tree boosting system. In: Proceedings of the 22nd ACM International Conference on Knowledge Discovery and Data Mining. ACM, New York. pp 785-794

Classical seasonal decomposition by moving averages. http://stat.ethz.ch/R-manual/R-devel/library/stats/html/ decompose.html. Accessed Nov 2017

Desalegn AA (2013) Assessment of drug use pattern using who prescribing indicators at Hawassa University teaching and referral hospital, South Ethiopia: A cross-sectional study. BMC Health Serv Res 13(1):170

Donker T, Wallinga J, Grundmann H (2010) Patient referral patterns and the spread of hospital-acquired infections through national health care networks. PLoS Comput Biol 6(3):1000715

Ellis MJ, Tao Y, Luo J, A'hern R, Evans DB, Bhatnagar AS, Chaudri Ross HA, von Kameke A, Miller WR, Smith I, et al. (2008) Outcome prediction for estrogen receptor-positive breast cancer based on postneoadjuvant endocrine therapy tumor characteristics. J Natl Cancer Inst 100(19):1380-1388

Fiterau M, Bhooshan S, Fries J, Bournhonesque C, Hicks J, Halilaj E, Ré C, Delp S (2017) Shortfuse: Biomedical time series representations in the presence of structured information. ArXiv preprint ArXiv:1705.04790. https://arxiv.org/abs/ 1705.04790

Genuer R, Poggi J-M, Tuleau-Malot C (2010) Variable selection using random forests. Pattern Recogn Lett 31(14):2225-2236

Hirsch JE (2005) An index to quantify an individual's scientific research output. PNAS 102(46):16569

Landon BE, Keating NL, Barnett ML, Onnela J-P, Paul S, O'Malley AJ, Keegan T, Christakis NA (2012) Variation in patient-sharing networks of physicians across the United States. JAMA 308(3):265-273

Lee BY, Song Y, Bartsch SM, Kim DS, Singh A, Avery TR, Brown ST, Yilmaz SL, Wong KF, Potter MA, et al. (2011) Long-term care facilities: Important participants of the acute care facility social network? PloS ONE 6(12):29342

Liaw Y-F (2009) On-treatment outcome prediction and adjustment during chronic hepatitis b therapy: Now and future. Antivir Ther 14(1):13-22

Lomi A, Mascia D, Vu DQ, Pallotti F, Conaldi G, Iwashyna TJ (2014) Quality of care and interhospital collaboration: A study of patient transfers in Italy. Med Care 52(5):407 
Mandl KD, Olson KL, Mines D, Liu C, Tian F (2014) Provider collaboration: Cohesion, constellations, and shared patients. J Gen Intern Med 29(11):1499-1505

NetworkX. http://networkx.github.io. Accessed Nov 2017

Page L, Brin S, Motwani R, Winograd T (1999) The pagerank citation ranking: Bringing order to the web. Technical report, Stanford InfoLab

Rombach P, Porter MA, Fowler JH, Mucha PJ (2017) Core-periphery structure in networks (revisited). SIAM Rev 59(3):619-646

Scikit-learn: Machine learning in Python. http://scikit-learn.org/stable/. Accessed Nov 2017

Shea D, Stuart B, Vasey J, Nag S (1999) Medicare physician referral patterns. Health Serv Res 34(1 Pt 2):331

Uddin S (2016) Exploring the impact of different multi-level measures of physician communities in patient-centric care networks on healthcare outcomes: A multi-level regression approach. Sci Rep 6:20222

Uddin S, Hamra J, Hossain L (2013) Mapping and modeling of physician collaboration network. Stat Med 32(20):3539-3551

Wu C-L, Wang F-T, Chiang Y-C, Chiu Y-F, Lin T-G, Fu L-F, Tsai T-L (2010) Unplanned emergency department revisits within 72 hours to a secondary teaching referral hospital in Taiwan. J Emerg Med 38(4):512-517

\section{Submit your manuscript to a SpringerOpen ${ }^{\circ}$} journal and benefit from:

- Convenient online submission

- Rigorous peer review

- Open access: articles freely available online

- High visibility within the field

- Retaining the copyright to your article

Submit your next manuscript at $\gg$ springeropen.com 